Chapter 18

\title{
Brainstem Gliomas
}

\author{
Zhiping Zhou and Mark M. Souweidane \\ Additional information is available at the end of the chapter \\ http://dx.doi.org/10.5772/53887
}

\section{Introduction}

Brainstem gliomas are a heterogeneous group of tumors occurring in the brainstem and cervicomedullary junction. They occur more often in children than in adults. Brainstem tumors, according to data prior to early 1980 s, represent $10-20 \%$ of all central nervous system neoplasms in the pediatric population [1-3]. Approximately $90 \%$ of brainstem tumors are gliomas in origin [4].

Diffuse intrinsic pontine glioma (DIPG) is the most common type representing approximately $80 \%$ of brainstem gliomas $[5,6]$. They have a dismal prognosis with a median survival of only a year. Their natural history is often compared to but worse than that of supratentorial glioblastoma seen primarily in the adult population. Nondiffuse brainstem gliomas, including focal, dorsal exophytic and cervicomedullary gliomas, are encountered less often and have a better prognosis. However, if untreated, they could also lead to progressive brainstem dysfunction and ultimately death.

\section{Epidemiology}

True incidence studies of brainstem gliomas are lacking. According to the Central Brain Tumor Registry of the United States, there were 400-450 cases of brainstem gliomas in children per year in the U.S. during 2004-2008, representing 10.7\% of primary central nervous system tumors [7]. This number is higher than those previously estimated for the period between 1970s-1990s [8]. The increase is thought primarily to be a result of increased detection by the use of magnetic resonance imaging (MRI) and better data reporting as opposed to a true increase in the incidence of these tumors. 
The most common age at diagnosis for DIPG is 5-9 years old with a median of 6.5 years, but cases have been seen in all age groups from infants to adults [5, 9]. Nondiffuse brainstem gliomas have roughly the same age distribution.

Neurofibromatosis type 1 (NF1) patients have higher incidence of brainstem gliomas. These tumors, whether diffuse or nondiffuse, appear to have a more benign course compared with tumors from patients without NF1 [10].

There does not appear to be a sex predilection in any of the brainstem tumor subgroups [5, 9]. Because of the low incidence and lack of diagnosis in less developed countries, there is not sufficient data to determine if there is a geographic propensity. No specific risk factors, either environmental or infectious, have been described to be related to the incidence of brainstem gliomas.

\section{Classification}

Brainstem gliomas are classified based on their location, focality and growth patterns. Classification systems were created using the current form of imaging available. Brainstem gliomas can be broadly categorized as diffuse intrinsic pontin glioma (DIPG) and nondiffuse brainstem gliomas. Nondiffuse brainstem gliomas can be further classified into three groups: focal gliomas (tectal and other locations), dorsal exophytic gliomas and cervicomedullary gliomas.

DIPG accounts for approximately $80 \%$ of brainstem gliomas in children [5, 6]. Surgical resection is not possible due to the location and infiltrative nature of the tumor. The standard of care for DIPG is radiation therapy. They have a median survival of about 10-12 months [6].

Focal gliomas account for about $5 \%$ of brainstem gliomas in children. They may be located anywhere within the brainstem but are typically not in the ventral pons. Most focal gliomas occur in the midbrain, especially the tectum of the midbrain. Focal gliomas in other areas of the brainstem, such as medulla, are less often seen. As a result, some discussions about focal gliomas may focus on tectal gliomas. Focal gliomas have well defined margins. They usually have an indolent course, and depending on location, are often amenable to surgical resection. The majority of tectal glioma patients have non-communicating hydrocephalus at initial presentation due to compression of cerebral aqueduct. Focal gliomas have a good prognosis [11].

Dorsal exophytic gliomas account for $10-15 \%$ of brainstem gliomas in children. They present insidiously with a long history of nonspecific headache and vomiting. They are amenable to surgical resection. These tumors have a good outcome after surgical resection [12].

Cervicomedullary gliomas account for $5-10 \%$ of brainstem gliomas in childrens. They arise from the lower medulla or the upper cervical spinal cord. They are amenable to surgical resection. Long-term tumor control can be achieved in most patients with surgical resection alone $[13,14]$. 


\section{Pathology and pathophysiology}

Brainstem gliomas are typically astrocytomas. Focal, dorsal exophytic and cervicomedullary gliomas are usually pilocytic astrocytoma (WHO grade I) and fibrillary astrocytoma (WHO grade II). Other low-grade gliomas with indolent growth such as ganglioglioma are also seen. DIPGs are typically anaplastic astrocytoma (WHO grade III) and glioblastoma multiforme (WHO grade IV). Growth of low-grade gliomas typically respect fiber tracts and pial borders. In contrast, high-grade gliomas grow and expand without respecting anatomic boundaries of these surrounding tissues.

$70-90 \%$ of DIPGs are anaplastic astrocytoma (WHO grade III) and glioblastoma multiforme (WHO grade IV) if biopsied before radiation therapy is given [15-17]. The remainders are mainly fibrillary astrocytoma (WHO grade II). At a later stage, invasion of adjacent levels of the brainstem and cerebellar peduncles is common. At autopsy, the vast majority of DIPGs are high-grade and more than 50\% have disseminated within the neuraxis [18].

Most focal gliomas are low-grade [11, 19]. Low-grade gliomas seen in this subgroup include pilocytic astrocytoma, fibrillary astrocytoma and ganglioglioma. Most tectal gliomas cause hydrocephalus at an early stage due to its compression on the cerebral aqueduct.

Dorsal exophytic gliomas arise from subependymal glial tissue. Over $90 \%$ of these tumors are pilocytic astrocytomas [20] that grow along the path of least resistance. As a result, most of the tumor extends into the fourth ventricle rather than infiltrating the brainstem ventrally. Tumors that extend laterally and/or ventrally into the brainstem are usually more aggressive and of higher grade on pathological examination.

Cervicomedullary gliomas arise from either the lower medulla or upper cervical spinal cord. Most of them are low-grade astrocytomas, but gangliogliomas and ependymomas are also seen [14, 21]. Tumors with epicenters in the upper cervical cord grow dorsally into the cisterna magna. Those with epicenters in the lower medulla grow centrifugally as focal nodules.

\section{Molecular genetics}

Partially because of the low incidence of brainstem gliomas, their molecular genetics is less studied compared with some more common primary central nervous system tumors. However, there are some significant advances in the last a few years concerning both DIPG and focal brainstem gliomas.

DIPGs are genetically complex and distinct from both adult and pediatric supratentorial high-grade gliomas (HGG). Recent evidence points to platelet-derived growth factor (PDGF) and its receptors (PDGFR) as among the major driving forces of tumorigenesis in the majority of cases [22-26]. Another type of growth factor receptors involved in DIPG is epidermal growth factor receptor (EGFR). Expression of EGFR was reported in the majority of high 
grade and less than $20 \%$ of low grade DIPG by immunohistochemistry staining [27]. The same study also reported that $25 \%$ of grade III and $50 \%$ of grade IV tumors had EGFR gene amplification. Recent data show that strong EGFR immunohistochemistry staining was seen in about $27 \%$ cases [23], lower than in the earlier report, and that amplification rate of the EGFR gene is also much lower (about 7-9\%) [23, 25]. Unlike the case in pediatric supratentorial HGG, CDKN2A deletion is non-existent in DIPG $[25,28]$ and amplification of CDK4 and CDK6 only occurs at a low rate [25]. Approximately 50\% of DIPG had p53 mutation [29, 30] and three groups reported loss of a region of $17 \mathrm{p}$ containing the p53 gene in $31 \%, 57 \%$ and $64 \%$ cases, respectively $[23,28,31]$. In approximately $50 \%$ of DIPG patients, allelic loss of a region of $10 \mathrm{q}$ where PTEN is located was observed [31-33].

There have been fewer molecular genetics studies of nondiffuse brainstem gliomas. Recently it was reported that BRAF gene duplication and mutation are often observed in pediatric pilocytic astrocytomas and fibrillary astrocytomas, including those located in the brainstem [34-36].

\section{Clinical presentation}

The clinical behavior and presentation of brainstem gliomas are highly variable, depending on the anatomic location and growth pattern of the tumor, age of the patient and certain other factors. Symptom evolution reflects aggressiveness of the tumor. Low-grade tumors typically present with an insidious course over several months of gradual symptom progression. By comparison, a shorter and more abrupt onset of symptoms is indicative of higher-grade tumors.

Nondiffuse gliomas are often low-grade lesions with expectedly slow growth. Symptoms are insidious in onset and may not be readily appreciated. Careful history taking, including comparison to photographs, may be necessary to evaluate subtle changes associated with cranial nerve dysfunction in these slow-growing tumors. DIPGs, in comparison, typically present with a short prodrome of symptoms (usually 1-2 months or shorter) [9].

In brainstem gliomas, the pattern of symptoms reflects the extent of the tumor. Diffusely growing tumors are associated with multiple and bilateral cranial neuropathies, long tract signs and ataxia. Hydrocephalus in these tumors is uncommon at initial presentation. Nondiffuse gliomas often present with isolated cranial neuropathies, signs and symptoms of increased intracranial pressure, and ataxia. Rarely, hemiparesis may be part of the clinical presentation.

Brainstem glioma patients can have a combination of the following at presentation.

Cranial neuropathies are common in most tumors of the brainstem. Simultaneous involvement of multiple cranial nerves is more frequently encountered with diffuse lesions. Focal lesions, in contrast, are often associated with more limited cranial nerve involvement. Upper brainstem involvement is often associated with oculomotor deficits, and lesions of the lower 
brainstem are frequently found to have lower cranial nerve involvement resulting in changes in voice, dysphagia and/or aspiration pneumonias.

Long tract signs, including motor dysfunction and hyperreflexia, are most often associated with diffusely growing tumors or focal tumors of the cervicomedullary junction. Long tract signs are often conspicuously absent with dorsal exophytic gliomas [12].

In brainstem glioma patients, ataxia can be a result of loss of proprioceptive sensation, $\mathrm{CN}$ VIII involvement and/or invasion of cerebellar peduncles by the tumor. Ataxia is frequently seen in DIPG patients.

Obstructive hydrocephalus is usually the first sign seen in focal tectal gliomas as a result of the tumor compressing cerebral aqueduct. Hydrocephalus and its associated signs and symptoms are also often seen in dorsal exophytic gliomas which grow dorsally into the fourth ventricle resulting in the obstruction of normal cerebrospinal fluid (CSF) flow.

Failure to thrive is commonly encountered in infants with tumors of the cervicomedullary junction due to lower cranial nerve dysfunction resulting in swallowing difficulties.

\subsection{Diffuse intrinsic pontine gliomas}

DIPG patients usually have an acute onset and present with a history of rapid deterioration of 1-2 months or even shorter. Physical examination reveals classic brainstem signs: cranial nerve deficits, long tract signs and ataxia, or combinations of the three. At least two of the triad need to be present along with MRI evidence to establish the diagnosis of DIPG.

Bilateral and multiple cranial nerve involvement is frequent in DIPG. Abducens (CN VI) palsy is the most frequent cranial neuropathy at presentation [5]. Facial palsy is also frequently seen at initial presentation. Involvement of CN VIII causes deadness and contributes to ataxia. Lower cranial nerve involvement causes aspiration, and difficulty in speech, swallowing and maintaining normal head positions.

These children often present with severe limb weakness and hyperreflexia, resulting in their difficulty to walk and sometimes even to sit. Long tract involvement also causes loss of tactile, nociceptive and proprioceptive sensations, causing numbness and contributing to ataxia.

In DIPG patients, both CN VIII involvement and loss of proprioceptive sensation contribute to ataxia. Invasion of cerebellar peduncles is also common in DIPG, which is another factor contributing to ataxia.

DIPG patients may also have other symptoms including headache, nausea and vomiting etc. indicating increased intracranial pressure from edema or hydrocephalus. Approximately $10 \%$ of DIPG patients have hydrocephalus at initial presentation [5].

\subsection{Focal gliomas}

Focal gliomas can occur anywhere in the brainstem, but usually not in the vental pons. Their clinical presentations depend on the location. 
Focal tectal gliomas have an insidious clinical course and often compress on cerebral aqueduct to cause a non-communicating hydrocephalus [11,37]. As a result the initial presentation is usually those signs reflecting increased intracranial pressure: headache, vomiting, diplopia and papilledema.

Focal gliomas in the midbrain tegmentum and the medulla are usually larger than tectal gliomas and presents with cranial nerve dysfunction, long tract signs and ataxia [11, 38].

\subsection{Dorsal exophytic gliomas}

Dorsal exophytic gliomas grow into the fourth ventricle and typically present with signs and symptoms of increased intracranial pressure including headache, nausea and/or vomiting, lethargy, downward gaze preference, changes in vision and cranial nerve palsies (including abducens palsy). Infants with these tumors may present primarily with failure to thrive. Long tract signs are rare with these tumors [12].

\subsection{Cervicomedullary gliomas}

Cervicomedullary gliomas usually have an indolent course because the majority of these tumors are slow growing low-grade lesions. Two presenting syndromes have been described based on the epicenter of these tumors [39]. Medullary syndrome often presents as a failure to thrive secondary to nausea, vomiting or dysphagia. Also seen with this syndrome are sleep apnea, dysarthria and recurrent upper respiratory tract infections. Cervical cord syndrome presents with chronic neck pain and/or progressive myelopathy with spasticity and weakness. Hydrocephalus is unusual in cervicomedullary gliomas.

\section{Imaging studies}

Diagnosis of brainstem gliomas is based on clinical presentations and imaging studies. MRI is the imaging method of choice because of its high resolution and ability to differentiate the natures of lesions (tumor vs. inflammation etc.), which is especially important in the diagnosis of DIPG. MRI enables identification of important anatomic features and localization of tumors with multi-planar views. It allows a precise evaluation of the growth pattern and accurate preoperative diagnosis in most cases. Both T1- and T2-weighted axial and sagittal sequences should be obtained for the basic studies. Other useful sequences include fluid attenuated inversion recovery (FLAIR), diffusion tensor imaging (DTI), gradient echo (GRE) and susceptibility weighted imaging (SWI). DTI tractography may be useful in evaluating the relationship between the tumor and white matter tracts [40], which can be a part of the preoperative planning for nondiffuse brainstem gliomas.

\subsection{Diffuse intrinsic pontine gliomas}

DIPGs are diagnosed with clinical presentation and magnetic resonance imaging. Since the publication of the Children's Cancer Group (CCG) report in 1993 [41], when a brainstem tu- 
mor presents with typical symptoms and radiological appearance, it is usually considered that these findings are diagnostic and a biopsy is rarely performed. If, however, the clinical or radiological presentation is not typical, where brainstem encephalitis or an inflammatory process of the pons cannot be ruled out, a stereotactic biopsy would be needed before initiating radiation therapy and/or chemotherapy.

DIPGs infiltrate widely. MRI scans show infiltrative expansion of the pons that is typically hypointense on T1-weighted, hyperintense on T2-weighted and FLAIR images, and has no significant dorsal exophytic component. Involvement of adjacent levels of the brainstem and/or cerebellar peduncles is common. Envelopment of the basilar artery is commonly present in DIPG showing the tumor growing ventrally outside of the boundary of the brainstem. Contrast enhancement with gadolinium is variable, with no enhancement being common [42]. Enhancement, if present, is usually patchy, without known prognostic significance. Neuraxis dissemination of DIPG can be seen on MRI in more than $50 \%$ of patients during progression [18]. In some cases the tumor may show atypical features including prominent enhancement and/or hypointense T2-weighted/ FLAIR signals.

Magnetic resonance spectroscopy (MRS) is helpful in differential diagnosis when the tumor shows atypical features. Increased Cho/NAA (choline/N-acetyl aspartate) and Cho/Cr (choline/creatine) ratios in DIPG are useful in differentiating it from brainstem encephalitis, demyelination and other inflammatory processes when clinical and MRI presentations are atypical $[43,44]$. However, the differentiating power of MRS is limited in NF1-associated DIPG [43].

\subsection{Focal gliomas}

Before the availability of computed tomography (CT) and MRI for the diagnosis of intracranial lesions, focal tectal gliomas could only be found in autopsy evaluations. In the 1950s, when CSF diversion procedures were not as universally successful as of today, Kernohan and Sayre stated that tectal gliomas were "in all probability the smallest tumors in the human body that lead to the death of the patient [45]."

Focal gliomas show as focal lesions on MRI. Focal tectal gliomas are usually isointense on MRI and show as thickened tectal plate. Tectal gliomas rarely enhance with gadolinium [11, 37]. Central calcification may be noted rarely [46]. Hydrocephalus is seen in the vast majority of tectal gliomas, causing symptoms for which the patients are seeking medical attention.

Focal gliomas in other areas of the brainstem are usually hypointense on T1-weighted and hyperintense on T2-weighted images. The margins are well defined in focal tegmentum and medullary gliomas, and most are more or less round or spherical. They are frequently cystic with an intense rim enhancement after intravenous contrast agent administration. Most midbrain focal gliomas show upward extension to the thalamus and downward extension to the pons. 


\subsection{Dorsal exophytic gliomas}

Dorsal exophytic gliomas typically erupt through the pia/ependyma early in their growth, so imaging will show that the bulk of tumor outside of the brainstem. Imaging of patients suspected of dorsal exophytic gliomas should include MRI studies with and without intravenous gadolinium of the brain to differentiate this type of tumor from tumors in the fourth ventricle such as primitive neuroectodermal tumors (PNETs). On MRI, dorsal exophytic gliomas appear as a dorsal exophytic expansion into the fourth ventricle or below the cerebellum. They usually show as sharply demarcated hypointense signals on T1-weighted and hyperintense on T2-weighted images. Bright homogeneous contrast enhancement and hydrocephalus are common MRI findings [20].

\subsection{Cervicomedullary gliomas}

Imaging of patients suspected cervicomedullary gliomas should include MRI studies with and without intravenous gadolinium of the brain and cervical spine to determine the extent of tumor growth and involvement.

Cervicomedullary gliomas arise from either the lower medulla or upper cervical spinal cord. Growth pattern of the tumor will give some hints as to their epicenters and what structures are being placed at risk by tumor growth. Tumors with epicenters in the upper cervical cord will have their rostral growth blocked by the decussating fibers of the sensory and motor pathways. Instead, the tumor curves dorsally to grow into the cisterna magna at the cervicomedullary junction. Tumors with epicenters in the lower medulla grow centrifugally as focal nodules pushing the surrounding fibers to their side [21].

Cervicomedullary gliomas appear as solid masses within the above-described areas. They are hypointense on T1-weighted and hyperintense on T2-weighted images. They typically enhance homogeneously upon gadolinium injection.

\section{Diagnosis and differential diagnosis}

Diagnosis of brainstem gliomas is based on clinical presentations and imaging studies and needs to address the location and nature of the lesion.

It is expected that the patient's clinical course, examination and imaging studies be in agreement. With nondiffuse brainstem gliomas this congruence often prompts surgical intervention (resection and/or relief of hydrocephalus), particularly for those located at the cervicomedullary junction or dorsal exophytic in growth.

Incongruence between presentation and clinical studies suggests the need for biopsy prior to definitive treatment. In cases with disagreement between the imaging findings and clinical course (i.e. rapidly progressive course with imaging consistent with a focal or low-grade lesion), frozen sections should be obtained at the time of surgery prior to progressing to an aggressive resection. These instances may be representative of a more malignant tumor in 
which case the potential for iatrogenic injury during resection outweighs the potential benefit of an extensive resection.

Differential diagnosis includes other tumors in the brainstem and adjacent regions and lesions of other natures. Other tumors include lymphoma, metastatic tumors, germinoma and nongerminomatous germ cell tumors, PNET, hemangioblastoma, lipoma, acoustic neuroma, etc. Some of these tumors typically arise from nearby regions and may encroach into the brainstem, e.g., primary intracranial germ cell tumors usually occur in the pineal region and can invade the brainstem. The majority of nongerminomatous germ cell tumors can be differentiated by testing for secreted markers in the blood such as $\alpha$-fetoprotein and $\beta$-human chorionic growth hormone. PNET occurring in the brainstem, even though relatively rare, can appear similar to DIPG or nondiffuse brainstem gliomas. Because of the difference in treatment options from those for brainstem gliomas, it should be carefully differentiated when suspected. Lesions of other natures include infectious, autoimmune, other inflammatory, vascular and metabolic lesions etc.

The role of biopsy in the diagnosis of DIPG remains intensely debated [47, 48]. Before the wide adoption of MRI in the diagnosis of brainstem gliomas, biopsy was performed to confirm diagnosis and provide information on prognosis. However, biopsy has been only performed infrequently since mid-1990s. Of note is that since the Children's Cancer Group (CCG) report in 1993 [41], MRI has superseded biopsy as the main method of diagnosing DIPG. As better methods to characterize tumors become available and more molecularly targeted therapies have become available or are under development in recent years, there are increasing calls to perform biopsy more frequently in clinically diagnosed DIPGs. Brainstem biopsy is safe with low morbidity and mortality rates [49-51]. Survey showed that parents of children with DIPG are generally amenable to biopsy, even if the results may not benefit the patient [52]. Various focus groups have been holding discussions on criteria of recommending biopsy. Some investigational protocols of DIPG have included biopsy and molecular characterization as mandatory components in stratifying subjects for experimental therapy.

\section{Treatment}

Treatment of brainstem gliomas includes management of pain, bulbar symptoms and motor impairment, relief of hydrocephalus and general support. Brainstem glioma patients should be cared for by a multidisciplinary team including neurosurgeons, non-surgical specialists and other therapists.

Surgical intervention is indicated for certain nondiffuse brainstem gliomas. Tectal gliomas are usually indolent and present with hydrocephalus. Hydrocephalus is relieved by endoscopic third ventriculostomy (ETV) or ventricular shunt placement. Early resection of tectal gliomas is controversial. Dorsal exophytic gliomas are the most amenable to surgical resection in brainstem gliomas. The goal is to remove the exophytic component, and when safe, followed by debulking of the intrinsic component. Surgical resection is typically recom- 
mended early for cervicomedullary gliomas. Subtotal resection can be safely performed in most cases.

Adjuvant therapy for brainstem gliomas depends on the tumor location, pathological diagnosis and extent of previous surgical resection. Radiation therapy is the standard of care for DIPG, but it is not recommended as an early treatment for nondiffuse brainstem gliomas if pathology shows a low-grade glioma. It is usually used in patients with high-grade pathology, upon clinical and imaging evidence of progression, or late in the disease course $[5,38]$. Stereotactic radiosurgery is also used in nondiffuse brainstem gliomas. Chemotherapy has limited use in the management of brainstem gliomas. It is mainly used for high-grade pathology or at late stage of the disease.

\subsection{Diffuse intrinsic pontine glioma}

Surgical resection of DIPG is not safe because of its location and infiltrative nature. Biopsy is not indicated for patients with typical clinical presentation and MRI findings. Hydrocephalus, if present, should be treated with either an ETV or a shunt. A histological confirmation might be needed if imaging or clinical history is not typical for a DIPG, or if an investigational protocol requests it.

The standard of care for DIPG is involved field external beam radiation therapy, typically delivered at $1.8 \mathrm{~Gy} /$ fraction for 30-33 fractions in the North America. Significant clinical improvement occurs in $85 \%$ of patients receiving radiation therapy, but the improvement is usually transient, lasting only 3-4 months, before progressive decline in clinical status returns [6].

Various chemotherapeutic regimens have been studied either alone or in combination with radiation therapy. However, up to date, no chemotherapeutic agent, either alone or in combination, or in conjunction with radiation therapy, has altered the natural history of DIPG. Many patients are enrolled in clinical trials of new agents or previously used agents in previously untested combinations.

\subsection{Focal gliomas}

Most tectal gliomas in children have indolent growth with few symptoms except those caused by hydrocephalus. Early resection of focal tectal gliomas is controversial and infrequently done. Often, these tumors are managed by relief of hydrocephalus, followed by clinical observation and periodic MRI scans $[53,54]$. Treatment of the hydrocephalus typically includes ETV to restore CSF flow. Shunting is an alternative or can be considered for patients who fail ETV. Surgical resection may be considered on rare occasions.

Focal gliomas in other areas of the brainstem, including tegmentum and medulla, are usually indicated for surgical resection. Tissue diagnosis before radical resection is indicated for lesions with atypical clinical and imaging findings. For those patients initially put on observation, when tumor progression is observed, biopsy or sometimes radical resection is indicated. Transventricular endoscopic biopsy may be performed with or without CSF diversion 
when the tumor is adjacent to a ventricle's walls [55]. Stereotactic biopsies may be considered for lesions that cannot be approached with endoscopy where accurate histological diagnosis is warranted but more aggressive surgical resection is deemed unsafe. Surgical resection of focal, well-circumscribed lesions provides not only a tissue diagnosis but also a prolonged event-free survival in many cases.

Conventional radiation therapy has demonstrated limited efficacy in patients with focal gliomas. Tumors with high-grade features should be treated with radiation therapy after recovery from surgery or when surgery is not indicated, while low-grade tumors may be observed and treated only if there is subsequent tumor progression [5, 38]. Implantation of iodine-129 (I-129) or iodine-125 (I-125) seeds has been used in some patients with acceptable tumor control. I-125 is an alternative to resection, external beam radiation therapy and chemotherapy in children with progressively symptomatic low-grade gliomas in deep and eloquent areas where high postoperative morbidity would be expected with open surgery [56]. Stereotactic radiosurgery has been used as an alternative means for managing focal brainstem gliomas [57]. Its use has been reported in patients with progressive tumor growth or worsening neurological deficits.

The role of chemotherapy has not been well evaluated for focal brainstem gliomas. In general, for tumors that total or subtotal resection has been performed on and subsequently progress, chemotherapy may be considered with or without radiation therapy. In children younger than 3 years old, chemotherapy may be used alone in attempts to delay the need for radiation therapy.

\subsection{Dorsal exophytic gliomas}

Dorsal exophytic gliomas are the group most amenable to surgical resection among brainstem gliomas and total or near total resection alone usually results in a good prognosis [12, 20]. Long-term event-free survival is expected when these tumors are simply shaven down to the level of the floor of the fourth ventricle. They should not be aggressively "chased" into the brainstem. In patients with findings consistent with an obstructive hydrocephalus who have not previously undergone a CSF diversion procedure (e.g. ETV or shunting), placement of an external ventricular drain may be considered. Tumor specimens should be sent early for frozen sections. A high-grade lesion is an indication to halt the surgery, as there is no proven long-term benefit to debulking aggressive lesions. Resection of the tumor takes place primarily within the fourth ventricle on the extra-axial part. In instances where there is a large intramedullary component to the tumor or if it is felt that damage to the functional tissues of the brainstem would be unavoidable, subtotal resection should be attempted. Some have advocated that resection carried to the level of the floor of the fourth ventricle confers a sufficient balance between disease control and preservation of neurological function by minimizing the risk of injuring functional brainstem tissues.

Radiation therapy is used for inoperable tumors or recurrent disease not amenable to re-resection $[5,38]$. Treatment primarily consists of conventional external beam radiation therapy with 30-33 daily fractions of $1.8 \mathrm{~Gy} /$ fraction [58-60]. The use of stereotactic radiation therapy 
and radiosurgery has also been examined in small cohorts. Chemotherapy has no proven efficacy in the treatment of dorsal exophytic gliomas [61].

\subsection{Cervicomedullary gliomas}

Cervicomedullary gliomas are treated in a fashion similar to that of an intramedullary spinal cord glioma or a focal glioma of the medulla depending on the location of the epicenter. Specimens should be sent early for frozen sections. Identification of a highgrade lesion is an indication to halt the surgery, as there is no proven long-term benefit to debulking aggressive lesions. Surgical resection should begin with internal debulking from the center to periphery.

As the majority of these tumors are low grade, surgery is typically the initial treatment modality. Radiation therapy should be reserved for advanced or recurrent diseases not amenable to surgical resection $[5,38]$. Treatment primarily consists of conventional fractionated external beam radiation given at $1.8 \mathrm{~Gy} /$ fraction for 30-33 fractions [58-60]. The use of stereotactic radiation therapy and radiosurgery has also been examined in small cohorts. Although a number of trials have examined the efficacy of chemotherapy in the treatment of DIPG, no role has been defined for its use in the treatment of cervicomedullary tumors [61].

\section{Outcomes}

The outcome of brainstem gliomas depends on the location and pathological grade of the tumor. Focal, dorsal exophytic and cervicomedullary gliomas typically have a fairly good prognosis. In contrast, the median survival of DIPG patients is only about one year despite numerous investigational therapies. In both DIPG and nondiffuse brainstem gliomas, patients with neurofibromatosis, older age, and longer duration of symptoms before diagnosis are thought to have a more favorable outcome [10].

\subsection{Diffuse intrinsic pontine gliomas}

External beam radiation therapy transiently improves the neurological condition in $85 \%$ of DIPG patients, but the disease usually progresses within 3-4 months. No treatment has been shown to be effective at the time of progression. Clinical deterioration is usually fast once progression is detected. Median survival is $10-12$ months. Only $10 \%$ of patients survive beyond two years after diagnosis [6]. Children under the age of 3 years, young adults over 18 and those with NF1 may have a better prognosis.

\subsection{Focal gliomas}

Lesions typical of tectal gliomas on imaging studies usually need treatment for hydrocephalus only and rarely progress over many years. Hydrocephalus can be well managed in tectal gliomas. ETV for tectal gliomas has a success rate exceeding $70 \%$ and as high as $90 \%$. Focal gliomas in other areas of the brainstem, especially if the pathology is pilocytic astrosytoma, 
can be cured by gross total resection but long-term follow-up is necessary. One study noted a 4-year progression-free survival rate of $94 \%$ and an overall 4 -year survival rate of $100 \%$ in 17 patients with focal midbrain gliomas, which included tectal gliomas [11]. Stereotactic radiosurgery may be an effective primary treatment or adjunct to open surgery for focal brainstem gliomas. After gamma knife radiosurgery, most patients improved or stabilized but some worsened [57].

\subsection{Dorsal exophytic gliomas}

Dorsal exophytic gliomas are amenable to surgical resection. Gross total or subtotal resection can be achieved in most cases. They have a good outcome after surgical resection. Among 18 patients who underwent surgery, 17 survived and only four showed evidence of radiological progression after a median follow-up of 113 months [12]. Subsequent progression may require re-resection or radiation therapy.

\subsection{Cervicomedullary gliomas}

Tumor control can be achieved by surgical resection in most patients with cervicomedullary gliomas. 5-year progression-free survival rate is $60 \%$ and overall 5-year survival rate about $90 \%$ after surgical resection alone [14].

\section{Recent advances and future directions}

Because of the dismal prognosis of DIPG and the lack of therapeutic efficacy of current chemotherapies, innovative approach is urgently needed for the disease. One promising drug delivery method that may improve the outcome of DIPG patients is convection-enhanced delivery (CED). Another recent advance is the molecular characterization of DIPG, which may provide guidance for developing new therapies.

\subsection{Convection-enhanced delivery}

The blood-brain barrier (BBB) presents a significant obstacle to achieving therapeutic concentrations of systemically delivered agents in brain tumors. Convection-enhanced delivery (CED) is a method of local delivery that bypasses the BBB [62]. CED is typically accomplished by inserting a small-bore cannula directly into a tumor followed by drug infusion through the cannula. Experimental studies have led to the recognition that CED can be characterized by several common features:

- Local drug concentration can exceed that achieved with systemic administration by several thousand times.

- Systemic exposure by efflux into the vasculature is minimal.

- Tissue penetration by CED is well beyond that achieved by other local delivery methods that rely on diffusion rather than bulk flow such as drug-impregnated polymers. 
- Distribution is preferentially along white matter tracts, a pattern reminiscent of glioma cell invasion.

- CED can be used to deliver macromolecules such as monoclonal antibodies or recombinant toxins, which is not possible by systemic administration in the brain parenchyma or tumor.

It is hypothesized that CED is ideally suited for the treatment of DIPG based on several features particular to this disease such as the tumor's relative compact growth pattern, its tendency to migrate along white matter fibers, the rarity of tumor related cysts and the lack of cavitary changes from radiation therapy.

The safety of CED in the brainstem has been well established in rodents and non-human primates [63-71]. The tolerability of several therapeutic agents delivered using CED into the brainstem has also been investigated and most of them proven to be safe at high concentrations [72-78]. This approach has been safely used on a limited clinical basis.

Although it is clear that certain limitations exist in the application of CED for the treatment of DIPG, currently existing preclinical data are sufficient to guide the design of clinical studies. Decisions in the design of these studies include the selection of an appropriate agent or agents, surgical technique, parameters of infusion, timing of treatment and methods of assessing distribution, local concentration, safety and therapeutic efficacy. These questions, however, should not be a barrier for clinical implementation, but rather as goals for early phase clinical studies. Based on current preclinical data, CED may serve as the basis for a new approach in the treatment of DIPG.

\subsection{Molecular characterization of diffuse intrinsic pontine gliomas}

A promising advance that may aid the development of therapeutic agents for the treatment of DIPG is the recent molecular characterization of this tumor. Four groups independently discovered that platelet-derived growth factor (PDGF) and its receptors (PDGFR) are amplified or over-expressed in the majority of these tumors [22-26]. Another type of growth factor receptors involved in DIPG is epidermal growth factor receptor (EGFR). Expression of EGFR was reported in the majority of high grade and less than $20 \%$ of low grade DIPG by immunohistochemistry staining [27]. The same study also reported that $25 \%$ of grade III and $50 \%$ of grade IV tumors had EGFR gene amplification. Recent data show that strong EGFR immunohistochemistry staining was seen in about $27 \%$ cases [23], lower than in the earlier report, and that amplification rate of the EGFR gene is also much lower (about 7-9\%) [23, 25]. Unlike the case in pediatric supratentorial HGG, CDKN2A deletion is non-existent in DIPG $[25,28]$ and amplification of CDK4 and CDK6 only occurs at a low rate [25]. Approximately $50 \%$ of DIPG had p53 mutation $[29,30]$ and three groups reported loss of a region of 17p containing the p53 gene in $31 \%, 57 \%$ and $64 \%$ cases, respectively [23, 28, 31]. In approximately $50 \%$ of DIPG patients, allelic loss of a region of $10 \mathrm{q}$ where PTEN is located was observed [31-33]. Like in adult malignant gliomas, the interleukin-13 receptor subtype IL-13R $\alpha 2$ is highly expressed in DIPG [79]. 
Some of these genetic aberrations have been the basis for DIPG animal models that could be powerful tools for studying the disease, e.g., Becher et al. designed a mouse model of DIPG based on PDGF overexpression [22]. Some of the molecular abnormalities have already been used as therapeutic targets. There are therapeutic monoclonal antibodies targeting PDGFR, namely IMC-3G3 (ImClone) and MEDI-575 (MedImmune), that need to be tested for efficacy in treating DIPG, preferably delivered through CED into the tumor since they are macromolecules unable to cross the BBB. There are numerous small molecule tyrosine kinase inhibitors with strong inhibitory effects on PDGFR and/or EGFR kinases, and inhibitors of downstream signaling molecules such as AKT and mTOR, that are undergoing clinical trials for the treatment of DIPG. There are also targeted recombinant toxins, e.g. IL13-PE38QQR, utilizing IL-13 as the targeting moiety to target tumor cells overexpressing IL-13 receptors. Even though biopsy of DIPG is far from being routine, when these molecularly targeted therapies come to clinical use, it would be ideal for the tumor to be pre-screened for specific targets of those agents.

\section{Conclusion}

Brainstem gliomas are a heterogeneous group of neoplasms with considerably diverse natural histories and prognoses. They are classified into four groups based on their location, focality and growth patterns. Diffuse intrinsic pontine gliomas (DIPG) account for approximately $80 \%$ of brainstem gliomas. The overwhelming majority of them is high grade. They are not resectable and the standard of care is involved field external beam radiation therapy. They have a dismal prognosis. The vast majority of focal, dorsal exophytic and cervicomedullary gliomas is low grade, often amenable to surgical resection and has a more favorable outcome.

Developing effective therapy for DIPG is so far frustrating as neither new schedules of radiation therapy nor chemotherapeutic regimens being studied have improved survival of these patients. As better molecular characterization methods become available, there are increasing calls to perform biopsy and tissue diagnosis in clinically diagnosed DIPGs. Recent molecular characterization of DIPG identified some genetic aberrations that could be used as effective therapeutic targets, including PDGF and PDGF receptors. There are both large and small molecule agents targeting these genetic aberrations that are either available or under development. However, most of these agents are unable to cross the blood-brain barrier (BBB) to reach the tumor. Convection-enhanced delivery (CED) is a drug delivery method to bypass the BBB. It has proven capability to deliver high local drug concentrations and the safety of its use in the brainstem has been established in small and large animals and a small number of patients. CED, combined with conventional chemotherapeutic drugs and novel targeted agents, has the potential to produce an effective treatment for DIPG. 


\section{Author details}

Zhiping Zhou ${ }^{1 *}$ and Mark M. Souweidane ${ }^{1,2}$

*Address all correspondence to: zhz2004@med.cornell.edu

1 Department of Neurological Surgery, Weill Medical College of Cornell University, New York, NY, USA

2 Department of Neurosurgery, Memorial Sloan-Kettering Cancer Center, New York, NY, USA

\section{References}

[1] Panitch, H.S. and B.O. Berg, Brain stem tumors of childhood and adolescence. Am J Dis Child, 1970. 119(6): p. 465-72.

[2] Farwell, J.R., G.J. Dohrmann, and J.T. Flannery, Central nervous system tumors in children. Cancer, 1977. 40(6): p. 3123-32.

[3] Albright, A.L., R.A. Price, and A.N. Guthkelch, Brain stem gliomas of children. A clinicopathological study. Cancer, 1983. 52(12): p. 2313-9.

[4] Pierre-Kahn, A., et al., Surgical management of brain-stem tumors in children: results and statistical analysis of 75 cases. J Neurosurg, 1993. 79(6): p. 845-52.

[5] Freeman, C.R. and J.P. Farmer, Pediatric brain stem gliomas: a review. Int J Radiat Oncol Biol Phys, 1998. 40(2): p. 265-71.

[6] Hargrave, D., U. Bartels, and E. Bouffet, Diffuse brainstem glioma in children: critical review of clinical trials. Lancet Oncol, 2006. 7(3): p. 241-8.

[7] Central Brain Tumor Registry of the United States, CBTRUS Statistical Report: Primary Brain and Central Nervous System Tumors Diagnosed in the United States in 2004-2008. 2012.

[8] Smith, M.A., et al., Trends in reported incidence of primary malignant brain tumors in children in the United States. J Natl Cancer Inst, 1998. 90(17): p. 1269-77.

[9] Kaplan, A.M., et al., Brainstem gliomas in children. A Children's Cancer Group review of 119 cases. Pediatr Neurosurg, 1996. 24(4): p. 185-92.

[10] Ullrich, N.J., et al., Brainstem lesions in neurofibromatosis type 1. Neurosurgery, 2007. 61(4): p. 762-6; discussion 766-7.

[11] Robertson, P.L., et al., Pediatric midbrain tumors: a benign subgroup of brainstem gliomas. Pediatr Neurosurg, 1995. 22(2): p. 65-73. 
[12] Pollack, I.F., et al., The long-term outcome after surgical treatment of dorsally exophytic brain-stem gliomas. J Neurosurg, 1993. 78(6): p. 859-63.

[13] Young Poussaint, T., et al., Cervicomedullary astrocytomas of childhood: clinical and imaging follow-up. Pediatr Radiol, 1999. 29(9): p. 662-8.

[14] Weiner, H.L., et al., Intra-axial tumors of the cervicomedullary junction: surgical results and long-term outcome. Pediatr Neurosurg, 1997. 27(1): p. 12-8.

[15] Wolff, J.E., et al., Subpopulations of malignant gliomas in pediatric patients: analysis of the HIT-GBM database. J Neurooncol, 2008. 87(2): p. 155-64.

[16] Wolff, J.E., et al., Intensive chemotherapy improves survival in pediatric high-grade glioma after gross total resection: results of the HIT-GBM-C protocol. Cancer, 2010. 116(3): p. 705-12.

[17] Cartmill, M. and J. Punt, Diffuse brain stem glioma. A review of stereotactic biopsies. Childs Nerv Syst, 1999. 15(5): p. 235-7; discussion 238.

[18] Sethi, R., et al., Prospective neuraxis MRI surveillance reveals a high risk of leptomeningeal dissemination in diffuse intrinsic pontine glioma. J Neurooncol, 2011. 102(1): p. 121-7.

[19] Hoffman, H.J., L. Becker, and M.A. Craven, A clinically and pathologically distinct group of benign brain stem gliomas. Neurosurgery, 1980. 7(3): p. 243-8.

[20] Khatib, Z.A., et al., Predominance of pilocytic histology in dorsally exophytic brain stem tumors. Pediatr Neurosurg, 1994. 20(1): p. 2-10.

[21] Epstein, F.J. and J.P. Farmer, Brain-stem glioma growth patterns. J Neurosurg, 1993. 78(3): p. 408-12.

[22] Becher, O.J., et al., Preclinical evaluation of radiation and perifosine in a genetically and histologically accurate model of brainstem glioma. Cancer Res, 2010. 70(6): p. 2548-57.

[23] Zarghooni, M., et al., Whole-genome profiling of pediatric diffuse intrinsic pontine gliomas highlights platelet-derived growth factor receptor alpha and poly (ADP-ribose) polymerase as potential therapeutic targets. J Clin Oncol, 2010. 28(8): p. 1337-44.

[24] Puget, S., et al., Mesenchymal transition and PDGFRA amplification/mutation are key distinct oncogenic events in pediatric diffuse intrinsic pontine gliomas. PLoS One, 2012. 7(2): p. e30313.

[25] Paugh, B.S., et al., Genome-wide analyses identify recurrent amplifications of receptor tyrosine kinases and cell-cycle regulatory genes in diffuse intrinsic pontine glioma. J Clin Oncol, 2011. 29(30): p. 3999-4006.

[26] Paugh, B.S., et al., Integrated molecular genetic profiling of pediatric high-grade gliomas reveals key differences with the adult disease. J Clin Oncol, 2010. 28(18): p. 3061-8.

[27] Gilbertson, R.J., et al., ERBB1 is amplified and overexpressed in high-grade diffusely infiltrative pediatric brain stem glioma. Clin Cancer Res, 2003. 9(10 Pt 1): p. 3620-4. 
[28] Barrow, J., et al., Homozygous loss of ADAM3A revealed by genome-wide analysis of pediatric high-grade glioma and diffuse intrinsic pontine gliomas. Neuro Oncol, 2011. 13(2): p. 212-22.

[29] Badhe, P.B., P.P. Chauhan, and N.K. Mehta, Brainstem gliomas--a clinicopathological study of 45 cases with p53 immunohistochemistry. Indian J Cancer, 2004. 41(4): p. 170-4.

[30] Zhang, S., et al., p53 gene mutations in pontine gliomas of juvenile onset. Biochem Biophys Res Commun, 1993. 196(2): p. 851-7.

[31] Louis, D.N., et al., Molecular genetics of pediatric brain stem gliomas. Application of PCR techniques to small and archival brain tumor specimens. J Neuropathol Exp Neurol, 1993. 52(5): p. 507-15.

[32] Cheng, Y. and $\mathrm{H}$. Wu, [Recent advances on molecular biology of diffuse astrocytoma]. Zhonghua Bing Li Xue Za Zhi, 1999. 28(3): p. 165-8.

[33] Cheng, Y., et al., Genetic alterations in pediatric high-grade astrocytomas. Hum Pathol, 1999. 30(11): p. 1284-90.

[34] Pfister, S., et al., BRAF gene duplication constitutes a mechanism of MAPK pathway activation in low-grade astrocytomas. J Clin Invest, 2008. 118(5): p. 1739-49.

[35] Schindler, G., et al., Analysis of BRAF V600E mutation in 1,320 nervous system tumors reveals high mutation frequencies in pleomorphic xanthoastrocytoma, ganglioglioma and extra-cerebellar pilocytic astrocytoma. Acta Neuropathol, 2011. 121(3): p. 397-405.

[36] Horbinski, C., et al., Association of molecular alterations, including BRAF, with biology and outcome in pilocytic astrocytomas. Acta Neuropathol, 2010. 119(5): p. 641-9.

[37] Bowers, D.C., et al., Tectal gliomas: natural history of an indolent lesion in pediatric patients. Pediatr Neurosurg, 2000. 32(1): p. 24-9.

[38] Ueoka, D.I., et al., Brainstem gliomas--retrospective analysis of 86 patients. J Neurol Sci, 2009. 281(1-2): p. 20-3.

[39] Epstein, F. and J. Wisoff, Intra-axial tumors of the cervicomedullary junction. J Neurosurg, 1987. 67(4): p. 483-7.

[40] Helton, K.J., et al., Diffusion tensor imaging of brainstem tumors: axonal degeneration of motor and sensory tracts. J Neurosurg Pediatr, 2008. 1(4): p. 270-6.

[41] Albright, A.L., et al., Magnetic resonance scans should replace biopsies for the diagnosis of diffuse brain stem gliomas: a report from the Children's Cancer Group. Neurosurgery, 1993. 33(6): p. 1026-9; discussion 1029-30.

[42] Mauffrey, C., Paediatric brainstem gliomas: prognostic factors and management. J Clin Neurosci, 2006. 13(4): p. 431-7.

[43] Porto, L., et al., Proton magnetic resonance spectroscopy in childhood brainstem lesions. Childs Nerv Syst, 2007. 23(3): p. 305-14. 
[44] Thakur, S.B., et al., Longitudinal MR spectroscopic imaging of pediatric diffuse pontine tumors to assess tumor aggression and progression. AJNR Am J Neuroradiol, 2006. 27(4): p. 806-9.

[45] Kernohan, G.W. and G.S. Sayre, Tumors of the central nervous system, in Atlas of Tumor Pathology: Section X1952, Armed Forces Institute of Pathology: Washington, DC.

[46] Daglioglu, E., O. Cataltepe, and N. Akalan, Tectal gliomas in children: the implications for natural history and management strategy. Pediatr Neurosurg, 2003. 38(5): p. 223-31.

[47] Leach, P.A., et al., Diffuse brainstem gliomas in children: should we or shouldn't we biopsy? Br J Neurosurg, 2008. 22(5): p. 619-24.

[48] Wilkinson, R. and J. Harris, Moral and legal reasons for altruism in the case of brainstem biopsy in diffuse glioma. Br J Neurosurg, 2008. 22(5): p. 617-8.

[49] Pincus, D.W., et al., Brainstem stereotactic biopsy sampling in children. J Neurosurg, 2006. 104(2 Suppl): p. 108-14.

[50] Pirotte, B.J., et al., Results of positron emission tomography guidance and reassessment of the utility of and indications for stereotactic biopsy in children with infiltrative brainstem tumors. J Neurosurg, 2007. 107(5 Suppl): p. 392-9.

[51] Roujeau, T., et al., Stereotactic biopsy of diffuse pontine lesions in children. J Neurosurg, 2007. 107(1 Suppl): p. 1-4.

[52] Bartels, U., et al., Proceedings of the diffuse intrinsic pontine glioma (DIPG) Toronto Think Tank: advancing basic and translational research and cooperation in DIPG. J Neurooncol, 2011. 105(1): p. 119-25.

[53] Walker, D.A., J.A. Punt, and M. Sokal, Clinical management of brain stem glioma. Arch Dis Child, 1999. 80(6): p. 558-64.

[54] Sandri, A., et al., Diffuse and focal brain stem tumors in childhood: prognostic factors and surgical outcome. Experience in a single institution. Childs Nerv Syst, 2006. 22(9): p. 1127-35.

[55] Ahn, E.S. and L. Goumnerova, Endoscopic biopsy of brain tumors in children: diagnostic success and utility in guiding treatment strategies. J Neurosurg Pediatr, 2010. 5(3): p. 255-62.

[56] Korinthenberg, R., et al., Long-term results of brachytherapy with temporary iodine-125 seeds in children with low-grade gliomas. Int J Radiat Oncol Biol Phys, 2011. 79(4): p. 1131-8.

[57] Yen, C.P., et al., Gamma knife surgery for focal brainstem gliomas. J Neurosurg, 2007. 106(1): p. 8-17.

[58] Schild, S.E., et al., The results of radiotherapy for brainstem tumors. J Neurooncol, 1998. 40(2): p. 171-7. 
[59] Skowronska-Gardas, A., Evaluation of radiotherapy for pediatric CNS tumors. Expert Rev Neurother, 2003. 3(4): p. 491-500.

[60] Hoffman, K.E. and T.I. Yock, Radiation therapy for pediatric central nervous system tumors. J Child Neurol, 2009. 24(11): p. 1387-96.

[61] Allen, J.C. and J. Siffert, Contemporary chemotherapy issues for children with brainstem gliomas. Pediatr Neurosurg, 1996. 24(2): p. 98-102.

[62] Bobo, R.H., et al., Convection-enhanced delivery of macromolecules in the brain. Proc Natl Acad Sci U S A, 1994. 91(6): p. 2076-80.

[63] Sandberg, D.I., M.A. Edgar, and M.M. Souweidane, Effect of hyperosmolar mannitol on convection-enhanced delivery into the rat brain stem. J Neurooncol, 2002. 58(3): p. 187-92.

[64] Sandberg, D.I., M.A. Edgar, and M.M. Souweidane, Convection-enhanced delivery into the rat brainstem. J Neurosurg, 2002. 96(5): p. 885-91.

[65] Occhiogrosso, G., et al., Prolonged convection-enhanced delivery into the rat brainstem. Neurosurgery, 2003. 52(2): p. 388-93; discussion 393-4.

[66] Croteau, D., et al., Real-time in vivo imaging of the convective distribution of a low-molecular-weight tracer. J Neurosurg, 2005. 102(1): p. 90-7.

[67] Krauze, M.T., et al., Safety of real-time convection-enhanced delivery of liposomes to primate brain: a long-term retrospective. Exp Neurol, 2008. 210(2): p. 638-44.

[68] Laske, D.W., et al., Chronic interstitial infusion of protein to primate brain: determination of drug distribution and clearance with single-photon emission computerized tomography imaging. J Neurosurg, 1997. 87(4): p. 586-94.

[69] Lonser, R.R., et al., Image-guided, direct convective delivery of glucocerebrosidase for neuronopathic Gaucher disease. Neurology, 2007. 68(4): p. 254-61.

[70] Lonser, R.R., et al., Successful and safe perfusion of the primate brainstem: in vivo magnetic resonance imaging of macromolecular distribution during infusion. J Neurosurg, 2002. 97(4): p. 905-13.

[71] Nguyen, T.T., et al., Convective distribution of macromolecules in the primate brain demonstrated using computerized tomography and magnetic resonance imaging. J Neurosurg, 2003. 98(3): p. 584-90.

[72] Souweidane, M.M., et al., Interstitial infusion of IL13-PE38QQR in the rat brain stem. J Neurooncol, 2004. 67(3): p. 287-93.

[73] Souweidane, M.M., et al., Interstitial infusion of carmustine in the rat brain stem with systemic administration of O6-benzylguanine. J Neurooncol, 2004. 67(3): p. 319-26.

[74] Luther, N., et al., Intraparenchymal and intratumoral interstitial infusion of anti-glioma monoclonal antibody 8H9. Neurosurgery, 2008. 63(6): p. 1166-74; discussion 1174. 
[75] Luther, N., et al., Interstitial infusion of glioma-targeted recombinant immunotoxin 8H9scFv-PE38. Mol Cancer Ther, 2010. 9(4): p. 1039-46.

[76] Murad, G.J., et al., Real-time, image-guided, convection-enhanced delivery of interleukin 13 bound to pseudomonas exotoxin. Clin Cancer Res, 2006. 12(10): p. 3145-51.

[77] Murad, G.J., et al., Image-guided convection-enhanced delivery of gemcitabine to the brainstem. J Neurosurg, 2007. 106(2): p. 351-6.

[78] Szerlip, N.J., et al., Real-time imaging of convection-enhanced delivery of viruses and virussized particles. J Neurosurg, 2007. 107(3): p. 560-7.

[79] Joshi, B.H., et al., Identification of interleukin-13 receptor alpha2 chain overexpression in situ in high-grade diffusely infiltrative pediatric brainstem glioma. Neuro Oncol, 2008. 10(3): p. 265-74. 
